\section{PERFIL DOS FREQUENTADORES DAS ACADEMIAS DE GINÁSTICA DE UM MUNICÍPIO DO RIO GRANDE DO SUL}

\author{
Profile of gym-goers from a municipality of Rio Grande do Sul \\ Perfil de los frecuentadores de los gimnasios de un municipio \\ de Rio Grande de Sul
}

Artigo Original

\section{RESUMO}

Objetivo: Traçar o perfil dos frequentadores das academias de ginástica do município de Canguçu-RS. Métodos: Estudo transversal, de caráter descritivo, com 177 frequentadores de academias de diversas modalidades. Utilizou-se um questionário estruturado, contendo 76 questões para mensurar variáveis sociodemograficas, antropométricas, nutricionais e comportamentais, além de informações relacionadas às praticas nas academias. Realizouse análise descritiva dos dados, com cálculo de médias \pm desvios-padrão para as variáveis contínuas e cálculo de proporções para as variáveis categóricas. Resultados: Participaram 177 frequentadores das academias na cidade de Canguçu-RS (30 12,5 anos; 70,0 $\pm 12,8 \mathrm{~kg}$; $1,69 \pm 0,1 \mathrm{~m})$. Com relação às variáveis comportamentais, a maioria nunca fumou $(71,8 \%)$, não bebia exageradamente $(89,9 \%)$ e era ativa no lazer $(87 \%)$. No tocante às variáveis nutricionais, 66,7\% tinham índice de massa corporal normal e a maioria não alcançava as recomendações de consumo diário de frutas, verduras e legumes, e não usava suplementos $(69,5 \%)$. No que diz respeito às variáveis relacionadas à academia, a maioria dos respondentes frequentava há mais de 12 meses $(56,5 \%)$, quatro ou mais vezes por semana $(52 \%)$, à noite $(52,5 \%)$, relatando que o principal motivo para ir à academia foi permanecer saudável (78\%). Os praticantes afirmaram satisfação com os objetivos alcançados $(60 \%)$ e praticavam a modalidade de musculação $(97,2 \%)$. Conclusão: A maioria dos frequentadores das academias era do sexo masculino, jovem, ativa no lazer, praticava a modalidade de musculação e estava satisfeita com os resultados buscados nas academias. Apesar disso, são necessárias estratégias de mudança de comportamento em relação ao consumo de frutas, verduras e legumes.

Descritores: Dieta; Academias de Ginástica; Atividade Física; Saúde.

\begin{abstract}
Objective: To determine the profile of gym-goers from the city of Canguçu, RS. Methods: Descriptive cross-sectional study conducted with 177 gym-goers in different modalities. We used a structured questionnaire containing 76 questions to measure sociodemographic, anthropometric, nutritional and behavioral variables, as well as information related to the activities at the gym. We conducted a descriptive analysis of data with estimation of means \pm standard deviations for continuous variables and estimation of proportions for categorical variables. Results: 177 gym-goers from the city of Canguçu, RS, participated in the study (30 12.5 years; $70.0 \pm 12.8 \mathrm{~kg} ; 1.69 \pm 0.1 \mathrm{~m})$. Regarding behavioral variables, most participants never smoked (71.8\%), did not drink excessively (89.9\%) and were active during leisure time (87\%). As to nutritional variables, $66.7 \%$ had normal body mass index and the majority did not meet the recommendations for daily consumption of fruit and vegetables and did not use dietary supplements (69.5\%). Regarding the variables related to the gym, most respondents have attended the gym for more than 12 months (56.5\%), four or more times a week (52\%), at night (52.5\%) and reported that the main reason for going to the gym was to be healthy (78\%). Respondents reported being satisfied with the goals achieved (60\%) and practiced weight training (97.2\%). Conclusion: Most gym-goers were young, males, active during leisure time, practiced weight training, and were satisfied with the results achieved at the gyms. Nevertheless, behavioral change strategies regarding the consumption of fruit and vegetables are necessary.
\end{abstract}

Descriptors: Diet; Gyms; Physical Activity; Health.
Recebido em: 16/03/2015

Revisado em: $21 / 04 / 2015$

Aceito em: 25/05/2015 


\section{RESUMEN}

Objetivo: Describir el perfil de los frecuentadores de los gimnasios del municipio de Canguçu-RS. Métodos: Estudio transversal, de carácter descriptivo con 177 frecuentadores de gimnasio de diversas modalidades. Se utilizó un cuestionario estructurado con 76 cuestiones para evaluar las variables sociodemograficas, antropométricas, nutricionales y de conducta, además de las informaciones relacionadas con las prácticas en los gimnasios. Se realizó un análisis descriptivo de los datos con el cálculo de las medias \pm desviación típica para las variables continuas y el cálculo de las proporciones para las variables categóricas. Resultados: Participaron 177 frecuentadores de

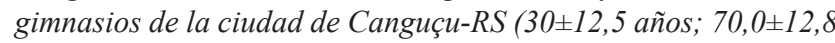
$\mathrm{kg} ; 1,69 \pm 0,1 \mathrm{~m})$. Respecto las variables de conducta, la mayoría nunca ha fumado (71,8\%), no bebía con exageración (89,9\%) $y$ era activa durante el ocio (87\%). Respecto las variables nutricionales, el $66,7 \%$ tenian el indice de masa corporal normal y la mayoría no alcanzaba las recomendaciones del consumo diario de frutas, verduras y legumbres y no consumía suplementos $(69,5 \%)$. Respecto las variables relacionadas con el gimnasio, la mayoría de los participantes frecuentaba desde hace más de 12 meses (56,5\%), cuatro o más veces a la semana $(52 \%)$, por la noche $(52,5 \%)$, relatando que el principal motivo para ir al gimnasio ha sido el hecho de permanecer saludable (78\%). Los practicantes afirmaron satisfacción con los objetivos alcanzados (60\%) y practicaban la modalidad de musculación (97,2\%). Conclusión: La mayoría de los frecuentadores de los gimnasios era del sexo masculino, joven, activos durante el ocio, practicaban la modalidad de musculación y estaban satisfechos con los resultados que buscaban en los gimnasios. A pesar de esto, son necesarias estrategias de cambios de conducta con respecto al consumo de frutas, verduras y legumbres.

Descriptores: Dieta; Centros de Acondicionamiento; Actividad Motora; Salud.

\section{INTRODUÇÃO}

As doenças crônicas não transmissíveis (DCNTs), em 2008, foram responsáveis por 36 milhões de óbitos, ou $63 \%$ do total de mortes no mundo ${ }^{(1)}$, e $74 \%$ do total de mortes no Brasil $^{(2)}$. A Organização Mundial da Saúde (OMS) projeta um aumento de $15 \%$ das mortes por DCNTs em todo mundo, entre os anos de 2010 e $2020^{(1)}$.

Dentre as DCNTs, as principais são as doenças cardiovasculares, diabetes, câncer e doenças respiratórias crônicas, responsáveis por $80 \%$ das mortes por DCNTs ${ }^{(1)}$. Essas enfermidades, na maioria das vezes, são causadas por quatro fatores de risco comportamentais, ou seja, modificáveis. São eles: uso do tabaco, dieta não saudável, consumo exagerado de álcool e sedentarismo ${ }^{(1)}$.

Os níveis de inatividade física no mundo são preocupantes. Segundo informações da OMS, em 2008, cerca de $31 \%$ dos adultos com idades entre 18 anos ou mais, globalmente, eram insuficientemente $\operatorname{ativos}^{(3)}$; e no mesmo ano, o sedentarismo foi identificado como o quarto principal fator de risco para a mortalidade global $(6 \%$ das mortes no mundo todo). Além disso, a OMS estima que a inatividade física será a principal causa para cerca de 21$25 \%$ dos cânceres de mama e de cólon, 27\% de diabetes e aproximadamente $30 \%$ da carga de doença isquêmica do $\operatorname{coração}^{(4)}$.

É notável o crescimento de estudos epidemiológicos relacionando atividade física como meio de promoção de saúde. Nas últimas décadas, estudos ${ }^{(5,6)}$ têm evidenciado, de forma consistente, que adequados níveis de atividade física diminuem o risco de desenvolvimento de doença arterial coronariana, diabetes, hipertensão e osteoporose.

Entre os espaços disponíveis para a prática de atividade física no Brasil, o número de academia de ginásticas, a partir da década de 1980, cresceu rapidamente no país, havendo uma grande procura, principalmente, pelos jovens ${ }^{(7)}$. Em 2012, o Conselho Federal de Educação Física (CONFEF) afirmou que o país alcançou a marca de 23.743 academias formalizadas, ficando atrás somente dos Estados Unidos. Segundo a Associação Brasileira de Academias, 6,7 milhões de pessoas frequentam esses estabelecimentos em busca, geralmente, de bem-estar, saúde, estética e, muitas vezes, de vida atlética ${ }^{(8)}$.

A literatura é escassa no que diz respeito ao perfil dos frequentadores das academias, o que torna inéditos os resultados do presente estudo, realizado em uma cidade do interior, com a maioria de sua população residindo na zona rural. A fim de preencher essa lacuna na literatura, bem como propiciar subsídios para os profissionais da área de educação física e para os proprietários, referentes ao tipo de público que frequenta as academias, o objetivo deste estudo foi traçar o perfil dos frequentadores das academias de ginástica do município de Canguçu-RS.

\section{MÉTODOS}

Realizou-se um estudo, no ano de 2014, na cidade de Canguçu-RS, do tipo transversal, de caráter descritivo. O município de Canguçu-RS é considerado o maior minifúndio da América Latina, com um total de 55.546 habitantes, dos quais por volta de $35 \%$ vivem na zona urbana $^{(9)}$.

A coleta dos dados teve início em outubro de 2013. Inicialmente, a cidade de Canguçu-RS teve suas academias de ginástica, devidamente registradas no $\mathrm{CREF} / \mathrm{RS}$, mapeadas pelos pesquisadores, a fim de se obter informações básicas dos estabelecimentos. O conceito de "academia de ginástica" utilizado no estudo foi um centro de atividade 
física, o qual disponibilizava inúmeras atividades, como: musculação, atividades aeróbicas, lutas, pilates etc., orientadas por profissionais de Educação Física.

A amostra foi formada por alunos que frequentassem as aulas há, no mínimo, um mês, de todas as modalidades oferecidas pelas academias que aceitaram participar da pesquisa. Fizeram parte quatro academias de ginástica e frequentadores das modalidades de musculação, ginásticas, lutas, pilates e atividades aquáticas. $\mathrm{O}$ estudo não previu cálculo para tamanho de amostra, visto que buscou entrevistar todos os frequentadores das academias.

A entrevista estruturada com os frequentadores foi realizada entre março e abril de 2014. Todos os frequentadores foram contatados e convidados a participar do estudo. Garantiu-se a todos o direito de recusa e a confidencialidade das informações a serem coletadas. Aqueles que concordaram em participar assinaram um termo de consentimento livre e esclarecido.

Utilizou-se para a coleta de dados um questionário contendo 76 questões, com o objetivo de mensurar variáveis sociodemográficas, antropométricas e nutricionais. O questionário incluiu perguntas criadas pelos pesquisadores e instrumentos previamente validados (relacionados aos comportamentos da prática atividade físicos, consumo exagerado de álcool e tabagismo). As variáveis sociodemográficas foram idade (em anos completos classificada em quatro categorias 18 a 29,30 a 39,40 a 49 e 50 anos ou mais); sexo (masculino ou feminino); estado civil (casado ou vivendo com companheiro, solteiro ou separado); cor (branca ou não branca); escolaridade (fundamental incompleto, fundamental completo, médio completo, médio incompleto, superior completo e superior incompleto); renda mensal familiar (até R\$ $1.000,00, \mathrm{R} \$$ $1.001,00$ a $\mathrm{R} \$ 2.000,00, \mathrm{R} \$ 2.001,00$ a $\mathrm{R} \$ 4.000,00$ e maior que $\mathrm{R} \$ 4.000,00)$. As variáveis nutricionais incluíram o índice de massa corporal - IMC (calculado a partir do peso e estatura referidos pelos entrevistados), hábitos alimentares e o consumo de suplementos.

Além disso, foram coletadas informações relacionadas às praticas nas academias (modalidade, frequência semanal, tempo/seção, objetivos da prática e nível de satisfação). $\mathrm{O}$ nível econômico foi definido a partir da renda mensal familiar e categorizado em classes (A-maior, B, C e D), de acordo com a Associação Brasileira de Empresas de Pesquisa ${ }^{(10)}$.

Adicionalmente, foram medidas as variáveis comportamentais, como o tabagismo e a ingestão de álcool, através de instrumentos propostos pela Organização Mundial da Saúde ${ }^{(11,12)}$. Com relação à variável ingestão de álcool, uma "dose" foi definida e explicada ao entrevistado como sendo equivalente ao consumo de $285 \mathrm{~mL}$ de cerveja, $120 \mathrm{~mL}$ de vinho ou, aproximadamente, $30 \mathrm{~mL}$ de destilados ${ }^{(12)}$.

Utilizou-se para a mensuração da prática de atividade física comportamental o Questionário Internacional de Atividades Físicas (IPAQ), versão longa e validada ${ }^{(13)}$. Os indivíduos que relataram prática semanal igual ou superior a 150 minutos foram considerados suficientemente ativos, seguindo as recomendações da $\mathrm{OMS}^{(14)}$.

Inicialmente, os dados foram transcritos para $\mathrm{o}$ Microsoft Office Excel 2007. Após a verificação de inexistência de erros, o banco de dados foi importado para o software estatístico STATA, versão 12 (StataCorp - Texas, USA). Realizou-se análise descritiva dos dados com cálculo de médias e desvios-padrão (DP) para as variáveis contínuas, e cálculo de proporções para as variáveis categóricas.

O estudo foi submetido ao Comitê de Ética e Pesquisa da Escola Superior de Educação Física da Universidade Federal de Pelotas e aprovado sob o $\mathrm{n}^{\circ}$. 31661014.0.000.5313, em obediência à Resolução 466/12.

\section{RESULTADOS}

Do total estimado de 400 alunos frequentadores das academias na cidade de Canguçu-RS, 177 foram entrevistados $(30 \pm 12,5$ anos; 70,0 $\pm 12,8 \mathrm{~kg} ; 1,69 \pm 0,1$ $\mathrm{m})$. A maior parte dos entrevistados possuía menos de 30 anos $(63,8 \% ; n=113)$. A Tabela I mostra que $92,7 \%(n=164)$ dos entrevistados tinham cor da pele branca (autorreferida), $49,7 \%(n=88)$ eram solteiros e $26 \%(n=42)$, estudantes. Com relação à escolaridade, a maioria possuía pelo menos ensino médio ou superior completo (55,3\%; $\mathrm{n}=98)$. Quanto à renda mensal familiar, a maioria dos entrevistados, $57,1 \%$ $(n=90)$ foi classificada nas classes econômicas B1 e B2. Do total de 177 entrevistados, a maioria $(97,2 \% ; n=172)$ praticava musculação e $15,8 \%(n=28)$ frequentavam aulas de ginástica, spinning e aeróbica.

A Tabela II apresenta os resultados relacionados às variáveis comportamentais. Quanto ao tabagismo, 71,8\% $(\mathrm{n}=127)$ da população relatou nunca ter fumado, e o hábito de fumar atualmente foi encontrado em $11,3 \%(n=20)$. Entre os homens que relataram ingerir bebida alcoólica atualmente, $8,8 \%(\mathrm{n}=10)$ ingeriram mais de cinco doses em uma mesma ocasião no último mês. Com relação às mulheres, a prevalência foi de $11,4 \%(\mathrm{n}=13)$ para a ingestão de mais de quatro doses em uma mesma ocasião no último mês. Analisando o comportamento de prática semanal de atividade física (atividade física total), constatou-se que a maioria da amostra, 94,9\%, era ativa. Quando analisados os domínios para prática de atividade física separadamente, os resultados variaram. Nos domínios do trabalho e do deslocamento, $59,3 \% \quad(n=105) \quad$ e $66,7 \% \quad(n=118), \quad$ respectivamente, mostraram-se insuficientemente ativos. Já nos domínios 
Tabela I - Características sociodemográficas dos praticantes de academias de ginástica. Canguçu-RS, 2014. (n=177)

\begin{tabular}{|c|c|c|}
\hline Variáveis & $\mathbf{n}$ & $\%$ \\
\hline \multicolumn{3}{|l|}{ Sexo } \\
\hline Masculino & 92 & 52,0 \\
\hline Feminino & 85 & 48,0 \\
\hline \multicolumn{3}{|l|}{ Idade (anos) } \\
\hline 18 a 29 anos & 113 & 63,8 \\
\hline 30 a 39 anos & 23 & 13,0 \\
\hline 40 a 49 anos & 27 & 25,3 \\
\hline 50 anos ou mais & 14 & 7,9 \\
\hline \multicolumn{3}{|l|}{ Cor da pele } \\
\hline Branca & 164 & 92,7 \\
\hline Preta/mulata/parda & 13 & 7,3 \\
\hline \multicolumn{3}{|l|}{ Estado civil } \\
\hline Casado (a) ou vive com companheiro (a) & 81 & 45,8 \\
\hline Solteiro (a) & 88 & 49,7 \\
\hline Separado (a) & 8 & 4,5 \\
\hline \multicolumn{3}{|l|}{ Escolaridade } \\
\hline Fundamental incompleto & 10 & 5,7 \\
\hline Fundamental completo & 12 & 6,8 \\
\hline Médio incompleto & 28 & 15,8 \\
\hline Médio completo & 62 & 35,0 \\
\hline Superior incompleto & 29 & 16,4 \\
\hline Superior completo & 36 & 20,3 \\
\hline \multicolumn{3}{|l|}{ Nível econômico (ABEP) } \\
\hline A (mais alto) & 5 & 2,8 \\
\hline $\mathrm{B} 1$ & 27 & 15,2 \\
\hline B2 & 63 & 35,6 \\
\hline $\mathrm{C} 1$ & 38 & 21,5 \\
\hline $\mathrm{C} 2$ & 41 & 23,2 \\
\hline $\mathrm{D}$ & 3 & 1,7 \\
\hline \multicolumn{3}{|l|}{ Modalidades que pratica na academia } \\
\hline Musculação & 172 & 97,2 \\
\hline Ginásticas & 28 & 15,8 \\
\hline Lutas & 13 & 7,3 \\
\hline Pilates & 3 & 1,7 \\
\hline Atividades aquáticas & 3 & 1,7 \\
\hline \multicolumn{3}{|l|}{ Ocupação } \\
\hline Estudante & 42 & 26,4 \\
\hline Comerciário(a) & 15 & 9,4 \\
\hline Empresário(a) & 13 & 8,2 \\
\hline Aposentado(a) & 8 & 5,0 \\
\hline Pedreiro & 7 & 4,4 \\
\hline Instrutor(a) de academia & 6 & 3,8 \\
\hline Professor(a) de ensino básico & 6 & 3,7 \\
\hline Técnico(a) em enfermagem & 5 & 3,1 \\
\hline Militar & 5 & 3,1 \\
\hline Outras & 70 & 22,9 \\
\hline
\end{tabular}

$\mathrm{ABEP}=$ Associação Brasileira de Empresas de Pesquisa 
lazer e domicílio, a maioria da amostra, $87 \%(n=154)$ e $67,8 \%(\mathrm{n}=120)$, respectivamente, mostrou-se ativa.

A Tabela III apresenta os resultados das variáveis nutricionais. Dos respondentes, $66,7 \%(\mathrm{n}=118)$ tiveram seu IMC classificado como normal e 41,8\% $(n=74)$ realizavam quatro refeições ao dia. Dos respondentes, 55,9\% ( $\mathrm{n}=98)$ consumiam frutas até quatro vezes por semana, e destes, $94,2 \%(n=162)$ afirmaram consumir até três porções ao dia. Com relação ao consumo de vegetais, $52,5 \%(\mathrm{n}=93)$ afirmaram consumir até quatro vezes por semana e $86,9 \%$ $(n=152)$ dos que consomem vegetais relataram consumir até três porções por dia.

No que diz respeito ao consumo de suplementos, $30,5 \%$ $(\mathrm{n}=54)$ afirmaram estar consumindo algum tipo de produto e $64,8 \%(n=35)$ consumiam há menos de 12 meses. Dentre os produtos mais consumidos, destacaram-se os suplementos compostos por proteínas $(83,3 \%, \mathrm{n}=45)$, creatina $(38,9 \%$; $\mathrm{n}=21)$, cafeína $(36,5 \% ; \mathrm{n}=19)$ e carboidratos $(24 \% ; \mathrm{n}=13)$.
Já em relação aos motivos para o consumo dos suplementos, destacaram-se o ganho de massa muscular $(90,7 \% ; n=49)$, melhorar o desempenho esportivo $(59,3 \% ; n=32)$ e permanecer saudável $(38 \% ; \mathrm{n}=21)$.

Analisando as variáveis relacionadas à utilização das academias de ginástica da cidade (Tabela IV), observouse que a maioria dos alunos $-56,5 \%(\mathrm{n}=100), 52 \%(\mathrm{n}=92)$ e $52,5 \%(n=93)$ - frequenta as academias há mais de um ano, quatro ou mais vezes por semana e no turno da noite, respectivamente. Com relação aos motivos que levaram essas pessoas a frequentarem as academias, destacaram-se: permanecer saudável $(78 \% ; \mathrm{n}=138)$, ganhar massa muscular $(74 \% ; n=129)$, ter mais energia/reduzir cansaço $(56,6 \%$; $n=99)$ e emagrecer $(50,3 \% ; n=89)$. Quanto à satisfação dos indivíduos com seus objetivos, a maioria afirmou estar satisfeita $(60,4 \% ; n=107)$, e no que se refere à qualidade dos aparelhos das academias, a maioria da amostra $(70,6 \%$; $\mathrm{n}=125$ ) os classificou como ótimo ou bom.

Tabela II - Frequência absoluta e relativa das variáveis comportamentais e de saúde dos praticantes de academias de ginástica. Canguçu-RS, 2014. (n=177)

\begin{tabular}{lcc}
\hline Variáveis & $\mathbf{n}$ & $\mathbf{\%}$ \\
\hline Fumantes & 127 & 71,8 \\
$\quad$ Nunca & 20 & 11,3 \\
Fumante atual & 30 & 16,9 \\
$\quad$ Ex-fumante & & \\
Ingestão de álcool de forma exagerada & 10 & 8,8 \\
$\quad$ Homens (mais de 5 doses) & 13 & 11,4 \\
$\quad$ Mulheres (mais de 4 doses) & 9 & 5,1 \\
Atividade física total & 168 & 94,9 \\
$\quad$ Insuficientemente ativos & & 59,3 \\
$\quad$ Ativos & 105 & 40,7 \\
Atividade física no trabalho & 72 & 13,0 \\
$\quad$ Insuficientemente ativos & & 87,0 \\
$\quad$ Ativos & 23 & 66,7 \\
Atividade física no lazer & 154 & 33,3 \\
Insuficientemente ativos & & 32,2 \\
Ativos & 118 & 67,8 \\
Atividade física no deslocamento & 59 & \\
$\quad$ Insuficientemente ativos & & \\
$\quad$ Ativos & 57 & \\
Atividade física no domicílio & 120 & \\
Insuficientemente ativos & & \\
Ativos & &
\end{tabular}


Tabela III - Frequência absoluta e relativa das variáveis nutricionais dos praticantes de academias de ginástica. Canguçu-RS, 2014. $(\mathrm{n}=177)$

\begin{tabular}{|c|c|c|}
\hline Variáveis & $\mathbf{n}$ & $\%$ \\
\hline \multicolumn{3}{|l|}{ Índice de massa corporal $\left(\mathrm{kg} / \mathrm{m}^{2}\right)$} \\
\hline Normal & 118 & 66,7 \\
\hline Sobrepeso & 46 & 26,0 \\
\hline Obesidade & 13 & 7,3 \\
\hline \multicolumn{3}{|l|}{ Número de refeições/dia } \\
\hline Até 3 & 35 & 19,8 \\
\hline 4 & 74 & 41,8 \\
\hline 5 ou mais & 68 & 38,4 \\
\hline \multicolumn{3}{|l|}{ Consumo de frutas/semana } \\
\hline Até 4 vezes & 98 & 55,9 \\
\hline 5 ou mais & 78 & 44,1 \\
\hline \multicolumn{3}{|l|}{ Porções de frutas consumidas/dia } \\
\hline Até 3 & 162 & 94,2 \\
\hline 4 ou mais & 10 & 5,8 \\
\hline \multicolumn{3}{|l|}{ Consumo de vegetais/semana } \\
\hline Até 4 vezes & 93 & 52,5 \\
\hline 5 ou mais & 84 & 47,5 \\
\hline \multicolumn{3}{|l|}{ Porcões de vegetais consumidos/dia } \\
\hline Até 3 & 152 & 86,9 \\
\hline 4 ou mais & 23 & 13,1 \\
\hline \multicolumn{3}{|l|}{ Usa suplemento } \\
\hline Não & 123 & 69,5 \\
\hline Sim & 54 & 30,5 \\
\hline \multicolumn{3}{|l|}{ Tempo de uso de suplemento (meses) } \\
\hline Até 12 & 35 & 64,8 \\
\hline 12 ou mais & 19 & 35,2 \\
\hline \multicolumn{3}{|l|}{ Polivitamínico/polimineral } \\
\hline Não & 42 & 77,8 \\
\hline Sim & 12 & 22,2 \\
\hline \multicolumn{3}{|l|}{ Proteínas } \\
\hline Não & 9 & 16,7 \\
\hline Sim & 45 & 83,3 \\
\hline \multicolumn{3}{|l|}{ Bebidas carboidratadas } \\
\hline Não & 41 & 75,9 \\
\hline Sim & 13 & 24,1 \\
\hline \multicolumn{3}{|l|}{ Cafeína } \\
\hline Não & 33 & 73,5 \\
\hline Sim & 19 & 36,5 \\
\hline \multicolumn{3}{|l|}{ Creatina } \\
\hline Não & 33 & 66,1 \\
\hline Sim & 21 & 38,9 \\
\hline \multicolumn{3}{|l|}{ Motivos para consumir suplementos } \\
\hline Permanecer saudável & 21 & 38,9 \\
\hline Melhorar desempenho esportivo & 32 & 59,3 \\
\hline Ter mais energia/reduzir cansaço & 16 & 29,6 \\
\hline Prevenir/tratar doenças/lesões & 16 & 29,6 \\
\hline Diminuir estresse & 7 & 13,0 \\
\hline Emagrecer & 11 & 20,4 \\
\hline Ganhar massa muscular & 49 & 90,7 \\
\hline Corrigir erros alimentares & 16 & 29,6 \\
\hline
\end{tabular}


Tabela IV - Frequência absoluta e relativa das variáveis relacionadas à utilização das academias de ginástica. Canguçu-RS, 2014. $(n=177)$

\begin{tabular}{lcc}
\hline Variáveis & $\mathbf{n}$ & $\mathbf{\%}$ \\
\hline Tempo de frequência à academia (meses) & 77 & 43,5 \\
Até 12 & 100 & 56,5 \\
12 ou mais & & \\
Frequência semanal à academia (vezes/semana) & 85 & 48,0 \\
Até 3 & 92 & 52,0 \\
4 ou mais & 52 & 29,4 \\
Turno que frequenta a academia* & 56 & 31,6 \\
Manhã & 93 & 52,5 \\
Tarde & & \\
Noite & 138 & 78,0 \\
Motivos para frequentar a academia & 75 & 42,4 \\
Permanecer saudável & 99 & 56,6 \\
Melhorar desempenho esportivo & 82 & 46,3 \\
Ter mais energia/reduzir cansaço & 87 & 49,2 \\
Prevenir/tratar doenças/lesões & 89 & 50,3 \\
Diminuir estresse & 129 & 74,6 \\
Emagrecer & & \\
Ganhar massa muscular & 28 & 15,8 \\
Satisfação com objetivos alcançados & 35 & 19,8 \\
Totalmente satisfeito & 107 & 60,4 \\
Muito satisfeito & 7 & 4,0 \\
Satisfeito & & \\
Insatisfeito & 21 & 11,9 \\
Qualidade dos aparelhos & 69 & 39,0 \\
Excelente & 56 & 31,6 \\
Ótima & 29 & 16,4 \\
Bom & 2 & 1,1 \\
Regular & & \\
Ruim & & \\
\hline
\end{tabular}

* O aluno pode frequentar mais de um turno por dia.

\section{DISCUSSÃO}

No presente estudo, a maioria dos participantes apresentou idade entre 18 e 29 anos, semelhante a estudo anterior $^{(15)}$. Uma possível explicação para o fato é que o público jovem prioriza a imagem corporal ${ }^{(7)}$, o que pode justificar a grande procura dessa parcela da população pelas academias.

Com relação à ocupação dos frequentadores, os estudantes aparecem em primeiro lugar, provavelmente pela faixa etária citada anteriormente. Outro ponto que deve ser ressaltado é o maior tempo disponível para a prática de atividade enquanto se é estudante, visto que a falta de tempo é um fator predominante para os altos índices de inatividade física mundialmente ${ }^{(16)}$. Um estudo que objetivou verificar as barreiras percebidas para a prática de musculação em adultos desistentes dessa modalidade ${ }^{(17)}$ observou que $55,2 \%$ dos adultos relataram a jornada de trabalho excessiva como a maior barreira para a prática de musculação.
No que diz respeito à escolaridade, classe social e etnia, os resultados do presente estudo vão ao encontro dos achados anteriores ${ }^{(18)}$, os quais sugerem uma tendência discriminatória do espaço das academias, onde somente pessoas com poder aquisitivo alto conseguem aderir a essas atividades.

Quanto às modalidades oferecidas pelas academias, a preferência apontada neste estudo foi também relatada em estudo anterior ${ }^{(19)}$, realizado com 159 alunos frequentadores das academias de ginástica de Belo Horizonte-MG. Parece bem estabelecido na literatura que as modalidades de academia relacionadas à capacidade física são as preferidas por praticantes do sexo masculino ${ }^{(20)}$.

Com relação ao nível de atividade física total e tempo de lazer, a maioria dos respondentes se mostrou suficientemente ativa. A literatura referente ao nível de atividade física de frequentadores de academias é escassa; isso pode ser explicado a partir do pressuposto de que essa população, por 
frequentar as academias, é naturalmente ativa ${ }^{(21)}$, pelo menos no tempo dedicado ao lazer. Porém, quando analisados os domínios de atividade física separadamente, elevados índices de ativos foram encontrados nos domínios "lazer" e "domiciliar", ao contrário dos domínios "deslocamento" e "trabalho". Esses dados vão ao encontro de um estudo populacional que mostrou grande prevalência de inativos no trabalho e deslocamento na população brasileira ${ }^{(22)}$.

No que diz respeito à variável nutricional IMC, um terço dos sujeitos foi classificado como estando em risco para sobrepeso e obesidade. Apesar do baixo custo e praticidade do IMC para determinar o estado nutricional de jovens e adultos, sua utilização para atletas e pessoas ativas pode gerar resultados imprecisos. Um estudo que comparou alguns indicadores antropométricos, como o IMC e dobras cutâneas, com homens frequentadores de academias mostrou que alguns indivíduos com a musculatura muito desenvolvida, mas com peso normal, foram classificados de forma equivocada com obesidade ou sobrepeso ${ }^{(23)}$. Nesse sentido, o percentual de sujeitos com sobrepeso e obesidade do presente estudo deve ser visto com cautela.

Com relação ao número de refeições realizadas por dia e o consumo de frutas, legumes e verduras, a maioria dos entrevistados no presente estudo não atendeu às recomendações nutricionais. Esses dados são preocupantes, pois em um estudo de base populacional ${ }^{(24)}$, foi investigado a relação entre a frequência alimentar, IMC e circunferência de cintura (CQ) de 1.355 homens e 1.645 mulheres, com idade entre 47 e 68 anos, que faziam parte de uma coorte de estudos em Malmo, na Suécia. Os resultados encontrados sugerem que uma frequência maior de refeições diárias foi inversamente associada com a probabilidade de obesidade geral e abdominal em homens, e associada positivamente a um estilo de vida saudável entre homens e mulheres. Por outro lado, a OMS recomenda o consumo de 400 gramas por dia de frutas e vegetais, ou cinco porções de 40 gramas por dia, para obtenção das quantidades necessárias de fitonutrientes, potássio e fibras, responsáveis pela diminuição do risco de doença coronariana, acidente vascular cerebral e hipertensão ${ }^{(25)}$.

Quanto ao consumo de suplementos nutricionais, quase um terço dos pesquisados afirmou estar utilizando algum tipo, sendo os produtos mais utilizados aqueles relacionados às proteínas ou aminoácidos. Um estudo anterior ${ }^{(19)}$ relatou prevalência maior de consumidores, $81,1 \%$, porém, os resultados corroboram com os encontrados na presente pesquisa no que diz respeito ao tipo de suplemento mais utilizado. Isso pode ser explicado pelo fato de que a maioria dos indivíduos que consome suplementos o faz visando um aumento de massa muscular, tendo em vista que a quantidade e o tempo de ingestão de proteínas são os principais fatores que regulam a síntese proteica, responsável, em parte, pelo desenvolvimento muscular ${ }^{(26)}$.

No que se refere à utilização das academias de ginástica, o presente estudo mostrou que a maioria frequentava há 12 meses ou mais, e por quatro ou mais dias por semana. Esses dados confirmam que a maioria dos praticantes que frequenta esses espaços tem condições financeiras e consciência da importância da atividade física, vai à academia quase todos os dias e mantém essa prática por um bom período de tempo, corroborando com um estudo ${ }^{(27)}$ realizado com adultos de ambos os sexos, frequentadores de uma academia de Rio Claro-SP.

No que diz respeito aos motivos relatados pelos alunos para frequentar as academias, estudos realizados em diversas regiões do Brasil observaram motivações para a prática de atividade física semelhantes às do presente estudo. Assim, pesquisas conduzidas no Rio Grande do Sul ${ }^{(7)}$ e no Paraná(15) encontraram estética ${ }^{(7)}$, saúde, aptidão física, disposição, atratividade e harmonia ${ }^{(15)}$ como os principais fatores motivadores para a prática.

Pode-se observar que o aspecto estético esteve entre os principais motivos de todas as pesquisas. No presente estudo, a estética pode ser interpretada nas opções "emagrecer" e "ganhar massa muscular". Isso sugere que, independentemente da região do país, e até mesmo em uma cidade do interior, a mídia pode influenciar as pessoas a procurarem as academias, uma vez que, diariamente, corpos perfeitos são exibidos na televisão, revistas e jornais, geralmente com baixo percentual de gordura e músculos aparentes, criando protótipos de beleza a serem seguidos. Um estudo de base populacional, realizado com 2.096 indivíduos com 10 ou mais anos de idade, tendo como objetivo avaliar a percepção populacional referente à influência da mídia sobre o estilo de vida, analisando quatro fatores comportamentais - atividade física, tabagismo, consumo de álcool e alimentação ${ }^{(28)}$-, observou que $80 \%$ dos indivíduos acreditavam que a mídia influenciava no estilo de vida individual. Porém, na presente pesquisa, o maior motivo para a prática foi permanecer saudável, o que pode sugerir uma maior preocupação e conscientização das pessoas em relação à prática de atividade física orientada e seus benefícios para a saúde.

Alguns aspectos importantes do presente estudo devem ser salientados. Deve-se ressaltar o ineditismo da pesquisa, por tratar-se de um estudo referente aos frequentadores de academias de uma pequena cidade. Adicionalmente, a logística cuidadosa e a utilização de instrumentos validados reforçam a fidedignidade dos dados. Por outro lado, limitações devem ser apontadas, especialmente no que diz respeito à variável IMC, calculada a partir dos valores de peso e estatura autorreferidos. Além disso, o IMC é o 
melhor indicador antropométrico a ser utilizado com atletas e pessoas fisicamente ativas, devido à possível confusão na interpretação dos resultados ${ }^{(24)}$.

\section{CONCLUSÃO}

A maioria dos frequentadores das academias era do sexo masculino, jovem, ativa no lazer, praticava a modalidade de musculação e estava satisfeita com os resultados buscados nas academias. Apesar disso, são necessárias estratégias de mudança de comportamento em relação ao consumo de frutas, verduras e legumes.

\section{REFERÊNCIAS}

1. World Health Organization - WHO. Global status report on noncommunicable diseases 2010. Geneva: WHO; 2011.

2. World Health Organization - WHO. Noncommunicable diseases country profiles 2011. Geneva: WHO; 2011.

3. World Health Organization - WHO. Global health risks: mortality and burden of disease attributable to selected major risks. Geneva: WHO; 2009.

4. World Health Organization - WHO. Global recommendations on physical activity for health. Geneva: WHO; 2010.

5. Wen CP, Way JPM, Tsai MK, Yang YC, Cheng TYD, Lee MC, et al. Minimum amount of physical activity for reduced mortality and extended life expectancy: a prospective cohort study. Lancet. 2011;378(9798):1244-53.

6. Lee IM, Shiroma EJ, Lobelo F, Puska P, Blair SN, Katzmarzyk PT. Effect of physical inactivity on major non-communicable diseases worldwide: an analysis of burden of disease and life expectancy. Lancet. 2012;380(9838):219-29.

7. Rufino VS, Soares LFS, Santos DL. Características de frequentadores de academias de ginástica do Rio Grande do Sul. Kinesis. 2000;22:57-68.

8. Souza P. Mercado fitness na região está aquecido. Conselho Federal de Educação Física 2012 [acesso em 2014 Fev 06]. Disponível em: http://www.confef.org. $\mathrm{br} /$ extra/clipping/view.asp?id $=420$

9. Instituto Brasileiro de Geografia e Estatística - IBGE. Diretoria de Pesquisas, Coordenação de População e Indicadores Sociais. NOTA 1: Estimativas da população residente com data de referência $1^{\circ}$ de julho de 2013 [acesso em 2014 Fev 06]. Disponível em: http://www.cidades.ibge.gov.br/xtras/temas.php?lang= $\&$ codmun $=430450 \&$ idtema $=119 \&$ search $=$ rio-grandedo-sul|cangucu|estimativa-da-populacao-2013

10. Associação Brasileira de Empresas de Pesquisa. Critério de classificação econômica Brasil. Associação Brasileira de Empresas de Pesquisa; 2011 [acesso em 2014 Maio 17]. Disponível em: http://www.abep.org

11. World Health Organization - WHO. Definitions of smoking. Global link. Geneva: WHO; 2003.

12. World Health Organization - WHO. Drinking and driving: a road safety manual for decision-makers and practitioners. Geneva: WHO; 2007.

13. Craig CL, Marshall AL, Sjostrom M, et al. International physical activity questionnaire: 12-country reliability and validity. Med Sci Sports Exerc. 2003;35(8):138195.

14. Haskell WL, Lee IM, Pate RR, Powell KE, Blair SN, Franklin BA, et al. Physical activity and public health: updated recommendation for adults from the American College of Sports Medicine and the American Heart Association. Med Sci Sports Exerc. 2007;39(8):142334.

15. Fermino RC, Pezzini MR, Reis RS. Motivos para prática de atividade física e imagem corporal em frequentadores de academia. Rev Bras Med Esporte. 2010;16(1):18-23.

16. Stutts WC. Physical activity determinants in adults. Perceived benefits, barriers, and self efficacy. AAOHN J. 2002;50(11):499-507.

17. Pinheiro KC, Silva DAS, Petroski EL. Barreiras percebidas para prática de musculação em adultos desistentes da modalidade. Rev Bras Ativ Fís Saúde. 2010;15(3):157-62.

18. Fontoura JL, Echeverria LC, Liberali R. Comparação do consumo de suplementos alimentares entre frequentadores de uma academia central e outra de periferia de uma cidade da região metropolitana de Porto Alegre/RS. Rev Bras Nutr Esportiva. 2009;3(15):24963.

19. Hallak A, Fabrini S, Peluzio MCG. Avaliação do consumo de suplementos nutricionais em academias da zona sul de Belo Horizonte, MG, Brasil. Rev Bras Nutr Esportiva. 2007;1(2):55-60.

20. Guimaraes Filho JCM, Moura DL, Antunes MM. Quando a beleza põe mesa: uma análise das condições de trabalho do profissional de educação física em megaacademias. Motrivivência. 2011;23(36):197-213. 
21. Delcor NS, Araujo TM, Reis EJ, Porto LA, Carvalho FM, Silva MO, et al. Labor and health conditions of private school teachers in Vitória da Conquista, Bahia, Brazil. Cad Saúde Pública. 2004;20(1):187-96.

22. Madeira MC, Siqueira FCV, Facchini LA, Da Silveira DS, Tomasi E, Thuné E, et al. Atividade física no deslocamento em adultos e idosos do Brasil: prevalências e fatores associados. Cad Saúde Pública. 2013; 29(1):165-74.

23. Ferreira LS, Honorato D, Stulbach T, Narciso P. Avaliação do IMC como indicativo de gordura corporal e comparação de indicadores antropométricos para determinação de risco cardiovascular em frequentadores de academia. Rev Bras Nutr Esportiva. 2013;7(42):324-32.

24. Holmaback I, Ericson U, Gullberg B, Wirfalt E. A high eating frequency is associated with an overall healthy lifestyle in middle-aged men and women and reduced likelihood of general and central obesity in men. $\mathrm{Br} \mathrm{J}$ Nutr. 2010;104(7):1065-73.

25. World Health Organization - WHO. Diet, nutrition, and the prevention of chronic diseases. Geneva: WHO; 2003.
26. Areta JL, Burke LM, Ross ML, Camera DM, West DWD, Broad EM, et al. Timing and distribution of protein ingestion during prolonged recovery from resistance exercise alters myofibrillar protein synthesis. J Physiol. 2013;591(9):2319-31.

27. Tahara AK, Schwartz GM, Silva KA. Aderência e manutenção da prática de exercícios em academias. Rev Bras Ciênc Mov. 2003;11(4):7-12.

28. Coll CDVN, Amorim TC, Hallal PC. Percepção de adolescentes e adultos referente à influência da mídia sobre o estilo de vida. Rev Bras Ativ Fís Saúde. 2010;15(2):105-10.

\section{Endereço para correspondência:}

Guilherme da Fonseca Vilela

Rua Luis de Camões, 625

Bairro: Três Vendas

CEP: 96055-630 - Pelotas - RS - Brasil

E-mail: gfviela@bol.com.br 